\section{Period analysis of the EEG by 6502-based microcomputer}

\author{
TYLER S. LORIG \\ Augustana College, Rock Island, Illinois
}

Period analysis has been a popular method of quantitative study of the EEG for many years (Brazier \& Finesinger, 1944). The technique involves the measurement of the duration of the major period of the EEG wave. These measurements may then be converted to frequencies so that the mean frequency of the EEG can be determined or the measurements may be used to construct a histogram of EEG period durations (Sharp, Smith, \& Surwillo, 1975). These techniques have been used successfully to detect differences in the EEG as the result of pharmacological treatments (Fink, 1975; Itil, 1975) and to study EEG development in children (Surwillo, 1975). Harner (1977) also suggested that this form of analysis produces results similar to spectral analysis when the EEG signal is relatively uniform. Unfortunately, computerized period analysis has been limited to mainframe and minicomputers (Legewie \& Probst, 1969; Sharp et al., 1975). The program described here is implemented on a 6502-based microcomputer and allows the user to collect up to eight channels of EEG period data for a user-selected number of time periods.

The program is highly interactive. The user may select the number of electrode pairs, the number of time periods, and the length of each time period prior to data collection. A data buffer is established that uses two pages of memory $(.5 \mathrm{~K})$ for each electrode pair during each time period. After data collection has been completed, the user may specify the starting frequency of EEG, the width of each frequency bin, and the number of frequency bins to be printed. The program then prints the amount of time (in seconds) that the major period of the EEG fell within each specified frequency range for each electrode pair (e.g., 8-10.9 Hz, $34.45 \mathrm{sec}$; 11-13.9 Hz, $21.59 \mathrm{sec} ; 14-16.9 \mathrm{~Hz}, 12.84 \mathrm{sec}$, etc.). This allows the user to produce a period histogram for each electrode pair within each interval of an experiment or demonstration. Good resolution of the EEG is available from 2 to $20 \mathrm{~Hz}$.

The data-collection system may use up to eight EEG amplifiers, a hardware-based EEG zero-crossing detector, and a 6502-based microcomputer that uses a peripheral interface device capable of reading a TTL level signal. The zero-crossing detector is a simple device that con-

Development of this program was supported by a grant to the author from the Augustana Faculty Research Committee. The author's address is: Department of Psychology, Augustana College, Rock Island, IL 61201. sists of various filters and a comparator integrated circuit. As the oscillating EEG signal crosses a zero point (either signal ground or a "moving" zero based on highly filtered EEG), the comparator outputs a square wave of the same duration as the EEG half-wave. This square wave is converted to a TTL level signal and is input into the computer's peripheral ports.

The period analysis program is written in BASIC and 6502 machine language. The machine language portion of the program operates in a "background" mode and samples the comparator output every $.001 \mathrm{sec}$. At the end of an EEG half-wave, the duration of the wave is accumulated into indexed bins. This produces a distribution of half-wave durations for each electrode pair and for each interval specified prior to data collection. The BASIC portion of the program passes data buffer addresses to the machine language program, depending upon elapsed time, starting address, and the number of electrode pairs. It also converts each distribution of half-wave durations into a distribution of EEG frequencies by doubling the half-wave duration.

Should the user not wish to make the assumption of EEG symmetry that is necessary for the conversion of a half-wave duration into an EEG frequency, the program is easily modified to produce an output of halfwave durations and the number of occurrences of each duration.

The program occupies $3 \mathrm{~K}$ of memory (not including the data buffer). It is based roughly on a technique described by Rayfield and Carney (1981). Although the program was originally developed on an AIM-65 microcomputer, it is easily adaptable to other 6502-based computers employing a peripheral interface device such as a versatile interface adapter integrated circuit chip (6522). A program listing and schematic of the zerocrossing detector are available from the author.

\section{REFERENCES}

Brazier, M. A. B., \& Finesinger, J. E. (1944). Characteristics of the normal electroencephalogram. Journal of Clinical Investigation, 23, 303-311.

FINK, M. (1975). Cerebral electrometry-Quantitative EEG applied to human psychopharmacology. In G. Dolce \& H. Kunkel (Eds.), CEAN-Computerized EEG analysis. Stuttgart: Fischer Verlag.

HARNer, R. N. (1977). EEG analysis in the time domain. In A. Rémond (Ed.), EEG informatics: A didactic review of methods and applications of EEG data processing. Amsterdam: Elsevier.

ITIL, T. (1975). Digital computer period analyzed EEG in psychiatry and psychopharmacology. In G. Dolce \& $\mathrm{H}$. Kunkel (Eds.), CEAN-Computerized EEG analysis. Stuttgart: Fischer Verlag.

Legewie, H., \& Probst, W. (1969). On-line analysis of the electroencephalogram with a small computer (period-amplitude analysis). Electroencephalography and Clinical Neurophysiology, 27, 533-535.

Rayfield, F., \& Carney, J. (1981). Controlling behavior experi- 
ments with BASIC on 6502-based microcomputers. Behavior Research Methods \& Instrumentation, 13, 735-740.

Sharp, F. H., Smith, G. W., \& Surwillow, W. W. (1975). Period analysis of the electroencephalogram with recordings of interval histograms of EEG half-wave durations. Psychophysiology, 12, 471-479.
SuRw ILlo, W. W. (1975). Interval histogram analysis of period of the electroencephalogram in relation to age during growth and development in normal children. Psychophysiology, 12, 506-512.

(Revision accepted for publication May 11, 1984.) 